\title{
A State Transition MIP Formulation for the Unit Commitment Problem
}

\author{
Semih Atakan, Guglielmo Lulli, Suvrajeet Sen, Member
}

\begin{abstract}
In this paper, we present the state-transition formulation for the unit commitment (UC) problem. This formulation uses new decision variables that capture the state transitions of the generators, instead of their on/off statuses. We show that this new approach produces a formulation which naturally includes valid inequalities, commonly used to strengthen other formulations. We demonstrate the performance of the statetransition formulation and observe that it leads to improved solution times especially in longer time-horizon instances. As an important consequence, the new formulation allows us to solve realistic instances in less than 12 minutes on an ordinary desktop PC, leading to a speed-up of a factor of almost two, in comparison to the nearest contender. Finally, we demonstrate the value of considering longer planning horizons in UC problems.
\end{abstract}

Index Terms-Mixed-integer linear programming, unit commitment

\section{INTRODUCTION}

Every day regional electricity networks deliver millions of kilowatt-hours of energy from generating units to consumers. These production requirements vary by season, day-of-the week, and hour. As a result, efficient scheduling of electricity production continues to attract significant attention from both industry and academia in the form of the so-called unit commitment (UC) problem. Such a model must recommend which generators to use, and how much they should produce so that demand over a planning horizon is met, while obeying certain operating rules, maintenance schedules, and in some instances, transmission capacity requirements. The goal is to obtain the most cost-effective operating schedule over a large set of generators, and ensure that the demand is completely fulfilled during the planning horizon.

With the liberalization of the energy industry, the introduction of energy market concepts and privatization, the role of the UC models has changed [1]. UC problems are solved by a variety of constituents of the electric power industry, each for a different purpose. A specific utility may solve the UC problem for the purposes of planning production within their delivery area. Their costs also helps them to place bids in the Independent System Operator (ISO) exchange market. On the other hand, the ISO solves its UC model to decide which bids to accept and to set prices that will be paid to the suppliers. These problems are much larger because they involve multiple suppliers, and with a number

S. Atakan \& S. Sen are with Daniel J. Epstein Department of Industrial and Systems Engineering, University of Southern California, Los Angeles, USA (e-mail: atakan@usc.edu; s.sen@usc.edu).

G. Lulli is with the Department of Management Science, Lancaster University, Lancaster, United Kingdom (e-mail: g.lulli@lancaster.ac.uk)

This research has been supported by the NSF (ECCS-1548847). of generating units in the order of thousands. Providing highquality solutions to these realistic problems is computationally very demanding but has the potential for significant reduction in costs. A 2011 report of the Federal Energy Regulatory Commission (FERC) suggests that savings approaching a $\$ 100$ million annually can be expected by replacing heuristics with methods that seek optimal solutions (see [2]). Consequently, developing solution methods that can achieve high-quality solutions in a short amount of time has been the focus of significant research over the last several decades.

Many optimization methods have been proposed to solve the UC problem. For example, we mention branch-andbound methods [3], dynamic programming approaches [4], Lagrangian relaxation methods [5], [6], and unit decommitment [7], among others. For a detailed review, the reader is referred to [8], [9]. In recent years, mixed-integer programming (MIP) has emerged as a popular tool for solving UC problems. A discussion on its merits and drawbacks (with respect to Lagrangian relaxation) were given in [10]. The popularity of MIP led to significant advances in the mathematical description of specific features of the UC problem. For instance, [11] have identified the convex hull for a minimum up/downtime polytope, [12] provided the convex hull of generation limits and this minimum up/downtime polytope, [13] provided strengthened inequalities of a ramping polytope, and [14] provided the convex hull of a two-period ramping polytope.

Nevertheless, it is well known that in general, mixed-integer programs are $\mathcal{N} \mathcal{P}$-hard, and solving problems of realistic size, involving thousands of generators, over several time periods, remains challenging. Moreover, the introduction of variable energy resources (e.g., wind and solar) leads to circumstances in which predictions from deterministic models are subject to significant errors. In order to accommodate such challenges, there have been recommendations that UC models should be solved using either the stochastic or the robust UC formulations (see, e.g., [15] and [16], respectively). In either case, the speed with which large scale deterministic UC models can be solved becomes important.

In this paper, we study MIP formulations of the UC problem with components that are of critical interest, although our study does not consider transmission networks. The contribution of our study is threefold. First, we develop a new formulation using a novel set of state-transition variables. These variables are named after their role in capturing the transition of generator states between consecutive time periods. They introduce a network sub-structure into the problem formulation. As observed originally by [17], and more recently by [18], this structure is much more amenable to LP-based 
solution methods, leading to faster solution times without modifying the underlying optimization methodologies. Second, we show that the use of state-transition variables naturally lead to certain facet-defining constraints in our problem-of-interest. This contrasts with the majority of other formulations available in the literature which often require the addition of further inequalities in order to generate the same strong (facet-defining) valid inequalities. Finally, we perform a computational study to demonstrate the behaviors of the new, and two relatively recent and well regarded benchmark formulations. This study sheds further light on the classes of instances for which the new formulation outperforms the contenders.

\section{FORMULATING PRODUCTION AND OPERATIONS IN UNIT COMMITMENT}

Beginning with the MIP formulation of [19], a plethora of mathematical formulations have been proposed in the literature to solve instances of the UC problem. These have extended the work of Garver in several directions. The mathematical modeling has been enriched with many additional aspects of the problem, and made it more realistic. For instance, several operational and technological restrictions have been included in the UC formulations, and significant efforts have been made in improving the formulation of operating costs. Other objectives have also been considered, such as the minimization of no load or turn off costs, maximization of social welfare, or maximization of the profit of generator companies (see [20]). In addition to refining the mathematical modeling, a lot of academic research in this field has been devoted to developing strong MIP formulations, so that real-scale instances of the problem can be handled with advanced MIP solvers.

As considered in [13] and [21], the core requirements of a UC problem involves the following components:

- minimum and maximum production restrictions,

- start up / shut down limits, and ramping restrictions,

- minimum up/downtime requirements,

- demand and reserve requirements (modeled as stand-by capacities)

- start up costs (defined as step-functions of the generators' idle times) and production costs (defined as piecewiselinear convex functions of the production amounts)

We adopt this core setup although other advanced requirements (e.g., transmission, power flow, line flow, and voltage limits, etc) are typically accommodated in realistic applications [22]. The purpose of our study will be to compare three alternative MIP formulations, which consider the above listed modeling considerations of a UC problem.

To begin our discussion, we present a prototypical MIP formulation of this UC model. Given a set of generators $\mathcal{G}$, and hourly discretized time periods $\mathcal{T}$, we introduce the following decision variables that are ubiquitous in the literature:

\footnotetext{
$x_{g, t}: \quad$ State variable ( 1 if $g$ is operational at time $t, 0$ otherwise),

$s_{g, t} / z_{g, t}:$ Start up / shut down variable (1 if $g$ is turned on / off at time $t, 0$ otherwise),

$p_{g, t}: \quad$ Amount of production by $g$ at time $t$.
}

The state variables are fundamental for the scheduling of generators. The start up / shut down variables are used to formulate the operating costs of the generators, whereas the production variables determine the dispatch amounts. In what follows, a vector of variables of the same type (say $x_{g, t}, \forall g \in \mathcal{G}, t \in \mathcal{T}$ ) will be typed in bold (say $\mathbf{x}$ ).

The objective of the mathematical formulation is to compute a power generation plan which satisfies production requirements along with operational constraints. The total operational costs include both the production and the start up costs. Both of these costs are nonlinear, in general. In our prototypical formulation, these costs are represented with the following (nonlinear) functions: $F_{g}(\cdot)$, for the start up costs, and $V_{g}(\cdot)$, for the production costs. The argument of the cost functions are respectively $i$ - and $j$-dimensional vectors $\mathbf{x}_{g,[t]}=\left(x_{g, t-i} \ldots x_{g, t}\right)$ and $\mathbf{p}_{g,[t]}=\left(p_{g, t-j} \ldots p_{g, t}\right)$, for some $i \geq 0$ and $j \geq 0$. This indicates the dependency of the costs, respectively, on the past states and the production levels of the generator.

A prototypical formulation is given below:

$$
\begin{array}{lll}
\min & \sum_{g \in \mathcal{G}} \sum_{t \in \mathcal{T}}\left(F_{g}\left(\mathbf{x}_{g,[t]}\right)+V_{g}\left(\mathbf{p}_{g,[t]}\right)\right) & \\
\text { s.t. } & x_{g, t}-x_{g, t-1}=s_{g, t}-z_{g, t} & \forall g \in \mathcal{G}, t \in \mathcal{T}, \\
& p_{g, t} \geq \underline{C}_{g} x_{g, t} & \forall g \in \mathcal{G}, t \in \mathcal{T}, \\
& p_{g, t} \leq \bar{C}_{g} x_{g, t} & \forall g \in \mathcal{G}, t \in \mathcal{T}, \\
& \sum_{g \in \mathcal{G}} p_{g, t} \geq d_{t} & \\
& (\mathbf{x}, \mathbf{s}, \mathbf{z}, \mathbf{p}) \in \mathcal{D} & \\
& (\mathbf{x}, \mathbf{s}, \mathbf{z}) \in\{0,1\}^{3|\mathcal{G}||\mathcal{T}|}, \mathbf{p} \geq \mathbf{0} .
\end{array}
$$

Above, $d_{t}$ is the demand for electricity at period $t, \bar{C}_{g}$ is the maximum generation capacity and $\underline{C}_{g}$ is the minimum required production amount when the generator is operational.

The constraints (1a) links the $s_{g, t}$ and $z_{g, t}$ variables to the state variables. Indeed, these variables are completely determined once the values of $\mathbf{x}$ are known. They are introduced exclusively to capture the transition of a generator between idle and operational state, and to formulate the operating costs of a generator. Constraints (1b) and (1c) model the lower and upper bounds, respectively. Constraints (1d) impose that the production levels must meet the demand for energy at any period. Finally, constraints (1e) state that a feasible solution must belong to the polyhedron $\mathcal{D}$, which will be described in detail in the following paragraphs.

The integrality requirements on the $(\mathbf{s}, \mathbf{z})$ variables can be relaxed without invalidating the formulation. This observation was exploited by [23] to formulate the UC problem with integrality restrictions on $\mathbf{x}$ alone. However, the assumed benefit of using considerably smaller number of binary variables does not necessarily lead to superior computational performance, as observed by [13]. This is due to improved formulations and more robust MIP solvers.

Before proceeding further, we define the following notation which will be used throughout this paper:
$\bar{R}_{g} / \underline{R}_{g}$ :
Ramp-up / ramp-down limit,
$\bar{S}_{g} / \underline{S}_{g}: \quad$ Start up / shut down limit,
$U T_{g} / D T_{g}$ : Minimum uptime / downtime limit. 
The data of a typical UC instance usually obey the following relations: $\bar{C}_{g} \geq \bar{R}_{g} \geq \bar{S}_{g} \geq \underline{C}_{g}>0$ and $\bar{C}_{g} \geq \underline{R}_{g} \geq \underline{S}_{g} \geq$ $\underline{C}_{g}>0, \forall g \in \mathcal{G}$. We assume $U T_{g} \geq 1$ and $D T_{g} \geq 1$ to avoid cases where the generator is simultaneously turned on and off.

\section{A. Formulating the components}

The majority of the UC formulations in the literature can be perceived as extensions of the prototypical formulation (1). These formulations differ in the way they define operational constraints, nonlinear objective functions, and production quantities. Nonetheless, the state of a generator is almost unanimously determined by $(\mathbf{x}, \mathbf{s}, \mathbf{z})$ variables, as first defined in the seminal work of [19]. In this section, we will identify all the components that go into modeling different considerations in a UC problem. In the next section, we will integrate them together into specific formulations, which correspond to the studies of [13] and [21]. In what follows, we will allow nonpositive indices of the decision variables to make it explicit that a solution of the problem might depend on the past states of the generators. In the actual implementation, we fix such variables to their realized values according to the available past data.

a) Formulating production quantities: An integral part of a UC formulation is the description of feasible production schedules that consider demand and operating reserve requirements along with minimum/maximum production restrictions. In the UC literature, the predominant choice for formulating production amounts has been through the $p_{g, t}$ variables. These variables are intuitive and simple, however, they may inadvertently introduce unneeded complexity into an MIP formulation. In particular, the presence of minimum production restrictions $1 \mathrm{~b}$ implicitly alters the continuous nature of these variables, leading to semi-continuous variables that must either equal 0 or lie within the range $\left[\underline{C}_{g}, \bar{C}_{g}\right]$. To avoid this, the following variables can be used in lieu of $p_{g, t}$ :

$p_{g, t}^{\prime}$ : The production amount beyond $\underline{C}_{g}$ provided by generator $g$ at time $t$.

This new variable only accounts for the variable portion of production. The fixed portion of production (i.e., the minimum production amount) is associated with the state variables as $\underline{C}_{g} x_{g, t}$. Combining these, we can form the linear mapping $p_{g, t} \rightarrow\left(p_{g, t}^{\prime}+\underline{C}_{g} x_{g, t}\right)$ which can be used to infer the total production amount. Moreover, after applying this mapping throughout the formulation, one can observe that the minimum production constraints are immediately satisfied. Consequently, $|\mathcal{G}| \times|\mathcal{T}|$ constraints in (1b) can be omitted from the formulation without sacrificing its fidelity.

The idea to treat the minimum and the variable production amounts separately was originally considered in [19]. Over the decades, this idea had been set aside, until the study of [21], who provided a modern look at this representation. Along with the use of $p_{g, t}^{\prime}$ variables, their study provides tighter descriptions of the production capacity restrictions which will be presented later in this section.

We continue with formulating the operating reserves. These requirements are stand-by capacities that must be kept ready to provide for unplanned outages of generating units. System operators use operating reserves to maintain system reliability and to ensure that the supply-demand balance is achieved seamlessly. [22] and [24] provide examples on how to formulate these requirements. To demonstrate, we introduce the following alternate sets of variables:

$\bar{p}_{g, t}$ : The maximum generation amount that $g$ can supply at time $t$,

$r_{g, t}$ : The generation amount that $g$ can supply at time $t$ for reserve requirements.

Both of these variables, by themselves, capture all the necessary information to formulate the reserve requirements. Indeed, the relation between these variables can be described with the mapping $\bar{p}_{g, t} \rightarrow\left(p_{g, t}+r_{g, t}\right)$.

In view of the discussion in this section, we provide two alternatives for formulating production, which respectively appeared in the state-of-the-art formulations of [13] (see constraints (2)-(3)) and [21] (see (4)-(6)). We begin with the former, where the $\left(p_{g, t}, \bar{p}_{g, t}\right)$ variables are used and the following production limits are imposed for all $g \in \mathcal{G}, t \in \mathcal{T}$ :

$$
\underline{C}_{g} x_{g, t} \leq p_{g, t} \leq \bar{p}_{g, t} \leq \bar{C}_{g} x_{g, t}+\left(\underline{S}_{g}-\bar{C}_{g}\right) z_{g, t+1} .
$$

The above constraints ensure that $(i)$ if the generator is idle (i.e., $x_{g, t}=0$ ), the production amounts cannot be positive, (ii) if the generator is operational (i.e., $x_{g, t}=1$ ), the minimum production requirements and capacity restrictions must be obeyed, and ( $i i i)$ if the generator is scheduled to be turned off in the next period (i.e., $z_{g, t+1}=1$ ), the maximum generation amount cannot exceed the shut down limit. For $t=|\mathcal{T}|$, the $z_{g, t+1}$ variable on the right-most inequality in (2) is assumed to be 0 . The $\bar{p}_{g, t}$ variables must also be smaller than the start up limit $\bar{S}_{g}$ whenever the generator is turned on at time $t$, however, this requirement will already be satisfied by the ramping inequalities presented in the next section. Finally, letting $\rho_{t}$ be the required reserve amount at time $t$, the following constraints make sure that the reserve requirements are fulfilled:

$$
\sum_{g \in \mathcal{G}} \bar{p}_{g, t} \geq d_{t}+\rho_{t} \quad \forall t \in \mathcal{T} .
$$

As an alternative for the above formulation of production, [21] utilize the $\left(p_{g, t}^{\prime}, r_{g, t}\right)$ variables. As previously stated, the minimum production constraints are redundant when $p_{g, t}^{\prime}$ variables are in use, therefore omitted. The following constraints provide a tight description of production limits:

$$
\begin{aligned}
& p_{g, t}^{\prime}+r_{g, t} \leq\left(\bar{C}_{g}-\underline{C}_{g}\right) x_{g, t}-\left(\bar{C}_{g}-\bar{S}_{g}\right) s_{g, t} \\
& \quad-\max \left(\bar{S}_{g}-\underline{S}_{g}, 0\right) z_{g, t+1} \forall g \in \mathcal{G}, t \in \mathcal{T},\left(U T_{g}=1\right) \\
& p_{g, t}^{\prime}+r_{g, t} \leq\left(\bar{C}_{g}-\underline{C}_{g}\right) x_{g, t}-\left(\bar{C}_{g}-\underline{S}_{g}\right) z_{g, t+1} \\
& \quad-\max \left(\underline{S}_{g}-\bar{S}_{g}, 0\right) s_{g, t} \forall g \in \mathcal{G}, t \in \mathcal{T},\left(U T_{g}=1\right) \\
& p_{g, t}^{\prime}+r_{g, t} \leq\left(\bar{C}_{g}-\underline{C}_{g}\right) x_{g, t}-\left(\bar{C}_{g}-\bar{S}_{g}\right) s_{g, t} \\
& \quad-\left(\bar{C}_{g}-\underline{S}_{g}\right) z_{g, t+1} \forall g \in \mathcal{G}, t \in \mathcal{T},\left(U T_{g}>1\right) .
\end{aligned}
$$

In the above inequalities, $z_{g, t+1}=0$ whenever $t=|\mathcal{T}|$. As shown in [12], these inequalities are facets of the convex hull of the polytope defined by generation limits and the minimum up/downtime constraints that will be presented shortly. 
With respect to constraints (1d), the use of $p_{g, t}^{\prime}$ variables necessitate an update in the demand constraints, as below:

$$
\sum_{g \in \mathcal{G}} p_{g, t}^{\prime}+\underline{C}_{g} x_{g, t} \geq d_{t} \quad \forall t \in \mathcal{T} .
$$

Finally, the following inequalities ensure that the reserve requirements can be fulfilled:

$$
\sum_{g \in \mathcal{G}} r_{g, t} \geq \rho_{t} \quad \forall t \in \mathcal{T} .
$$

b) Formulating operational and technological restrictions: In order to compute realistic production schedules, the MIP formulations must take into account the physical limitations of the components of the power systems. Among these limitations, some of the most essential ones are the minimum up/downtime and ramping restrictions. The minimum up/downtime restrictions ensure that the on/off status of the generators do not change rapidly. Frequent state transitions have several adverse consequences including (i) increased operator stress, (ii) diminished generator life, and (iii) increased emission of pollutants during transient periods (see [25]). Such restrictions are quite practical and are included in many commercial tools. To formulate these restrictions, several constraints were proposed in [24], [25], [26]. Following [11], the minimum uptime and downtime constraints are best formulated using the following constraints:

$$
\begin{array}{rl}
\sum_{i=t-U T_{g}+1}^{t} s_{g, i} \leq x_{g, t} & g \in \mathcal{G}, t \in \mathcal{T}, \\
\sum_{i=t-D T_{g}+1}^{t} s_{g, i} \leq 1-x_{g, t-D T_{g}} & g \in \mathcal{G}, t \in \mathcal{T} .
\end{array}
$$

Observe that when the generator $g$ is operational at time $t$, the right-hand side of constraint $(7 \mathrm{a})$ is set to 1 . In this case, the generator may have been turned on at most once in the last $U T_{g}$ periods (due to minimum uptime restrictions). On the other hand, if it is idle at time $t$, it could not have been turned on in the last $U T_{g}$ time periods, as otherwise it should be operational at time $t$. Constraint $(7 \mathrm{~b})$ is just a rewritten version of a similar constraint for the downtime requirements. It has been widely observed that these turn on/off inequalities significantly outperform its contenders [13]. Indeed, [11] showed that constraints (7) define facets of the polytope defined by the minimum up/downtime constraints.

The ramping restrictions limit the maximum change in production between consecutive periods and ensure that the generation requirements can be matched by the electricity production without exceeding the generator limitations over extended periods of time. A basic representation of these ramping restrictions, in the space of $p_{g, t}^{\prime}$ and $r_{g, t}$ variables, appears below:

$$
\begin{aligned}
p_{g, t}^{\prime}+r_{g, t}-p_{g, t-1}^{\prime} & \leq \bar{R}_{g} \quad \forall g \in \mathcal{G}, t \in \mathcal{T}, \\
p_{g, t-1}^{\prime}-p_{g, t}^{\prime} & \leq \underline{R}_{g} \quad \forall g \in \mathcal{G}, t \in \mathcal{T} .
\end{aligned}
$$

The above inequalities, by themselves, do not take into account the start up and shut down rates of the generators. Indeed, certain generation limit constraints (such as (4)) must be used in conjunction with $(8)$ to make sure these restrictions are also satisfied.
The ramping inequalities may also be written in the space of $p_{g, t}$ and $\bar{p}_{g, t}$ variables. Below, we give the formulation of ramping restrictions that appeared in [13].

$$
\begin{aligned}
\bar{p}_{g, t}-p_{g, t-1} \leq \bar{S}_{g} s_{g, t}+\bar{R}_{g} x_{g, t-1} & \forall g \in \mathcal{G}, t \in \mathcal{T}, \\
p_{g, t-1}-p_{g, t} \leq \underline{S}_{g} z_{g, t}+\underline{R}_{g} x_{g, t} & \forall g \in \mathcal{G}, t \in \mathcal{T} .
\end{aligned}
$$

Constraint 9a ensures that generator $g$ cannot ramp up more than $\bar{S}_{g}$ if it has just been turned on, or $\bar{R}_{g}$ if it remains on at time $t$. Similarly, constraint $9 \mathrm{~b}$ limits the decrease in the power output by $\underline{R}_{g}$ at any time that the generator remains operational. If the plant is turned off at $t\left(x_{g, t}=0\right)$, then the output of the generator cannot be larger than $\underline{S}_{g}$ to obey the shut down limits. To account for the reserve requirements, constraint $9 \mathrm{a}$ ) can be modified as follows:

Observe that (9a) cannot be tight if the generator is turned off at time $t\left(\bar{p}_{g, t}=0\right)$ because $-p_{g, t-1} \leq-\underline{C}_{g}<0$, but the right-hand-side is $\bar{R}_{g}>0$. A similar argument also applies to (9b) when the generator is turned on at time $t$. In fact, these (along with many others) were the motivation behind studying tighter ramping constraints and valid inequalities in [13], [14], [27], [28]. In particular, [14] provided the following strengthened ramping constraints:

$$
\begin{aligned}
p_{g, t}- & p_{g, t-1} \leq\left(\bar{S}_{g}-\bar{R}_{g}-\underline{C}_{g}\right) s_{g, t} \\
& +\left(\bar{R}_{g}+\underline{C}_{g}\right) x_{g, t}-\underline{C}_{g} x_{g, t-1} \quad \forall g \in \mathcal{G}, t \in \mathcal{T}, \\
p_{g, t-1}-p_{g, t} & \leq\left(\underline{S}_{g}-\underline{R}_{g}-\underline{C}_{g}\right) z_{g, t} \\
& +\left(\underline{R}_{g}+\underline{C}_{g}\right) x_{g, t-1}-\underline{C}_{g} x_{g, t} \quad \forall g \in \mathcal{G}, t \in \mathcal{T} .
\end{aligned}
$$

These inequalities were proved to be facet-defining for the two-period ramp-up and ramp-down polytopes (i.e., the polytopes of the UC problem that are limited to two consecutive periods and consider only the ramp-up and ramp-down constraints, respectively).

c) Linearization of the objective function: The nonlinear operating cost functions in the objective of a UC problem are typically approximated with piecewise-linear convex functions. We begin with the linearization of the start up costs. For a generator $g$, we use the set $\mathcal{I}_{g}=\left(t_{g}^{1} \ldots t_{g}^{\left|\mathcal{I}_{g}\right|}\right) \subseteq \mathcal{T}$ to denote the set of idle periods after which the cost incurred to turn on a generator changes. The start up costs are given by $f c_{g}^{\tau}$ where $\tau \in \mathcal{I}_{g}$. These costs are assumed to obey $f c_{g}^{|\mathcal{I} g|} \geq \ldots \geq f c_{g}^{1}$, $\forall g \in \mathcal{G}$, indicating that the start up costs tend to increase as the idle time of the generators grow. Accordingly, a generator that has been idle for 1 to $t_{g}^{1}-1$ periods will incur a start up cost of $f c_{g}^{1}, t_{g}^{2}$ to $t_{g}^{3}-1$ periods will incur $f c_{g}^{2}$, and so forth. It is easy to see that the start up costs are determined by a step-function. This function can be incorporated into an MIP formulation using the approaches in [29] and [21], where the former linearizes it and the latter partitions its domain. These approaches respectively require the following variables:

$f_{g, t}: \quad$ Incurred start up cost for generator $g$ at time $t$,

$\delta_{g, t, \tau}$ : Start up cost selection variable (1 if $g$ must incur the start up cost $f c_{g}^{\tau}$ at time $t, 0$ otherwise).

Using the $f_{g, t}$ variables, [29] determine the cost of turning on a generator with the following start up cost constraints:

$$
f_{g, t} \geq f c_{g}^{\tau}\left(x_{g, t}-\sum_{i=1}^{t_{g}^{\tau}} x_{g, t-i}\right) \forall g \in \mathcal{G}, t \in \mathcal{T}, \tau \in \mathcal{I}_{g} .
$$


To minimize the total start up costs, the objective must then contain the sum of all $f_{g, t}$ variables. Alternatively, the approach in [21] formulates the start up costs as follows:

$$
\begin{aligned}
& \delta_{g, t, \tau} \leq \sum_{i=t_{g}^{\tau}}^{t_{g}^{\tau+1}-1} z_{t-i} \quad \forall g \in \mathcal{G}, t \in\left(t_{g}^{\tau+1} \ldots|\mathcal{T}|\right), \\
& \tau \in \mathcal{I}_{g} \backslash\left\{1 \ldots D T_{g}-1, t_{g}^{\left|\mathcal{I}_{g}\right|}\right\}, \\
& \sum_{\tau=1}^{\left|\mathcal{I}_{g}\right|} \delta_{g, t, \tau}=s_{g, t} \quad \forall g \in \mathcal{G}, t \in \mathcal{T} .
\end{aligned}
$$

Constraints (12) ensure that a single and correct start up type is selected based on how long the unit has been idle. Notice that (12a) need not be defined for $\tau<D T_{g}$ (since an idle generator must obey the minimum downtime restrictions) and for $\tau=$ $t_{g}^{\left|\mathcal{I}_{g}\right|}$ (as it will be redundant due to $12 \mathrm{~b}$ ). The integrality restrictions on $\delta_{g, t, \tau}$ need not be enforced provided that the start up costs are monotonically increasing with the number of periods the generator remains idle.

In general, the production costs are formulated with piecewise-linear convex functions, as the marginal cost of production increases with increasing levels of production. We define a set of production amounts $p_{g}^{\kappa}$ (with $\kappa \in\left\{1 \ldots \kappa_{g}^{\max }\right\}$ ) at which the incurred unit cost of production for generator $g$ changes. These production levels can be interpreted as the breakpoints where the slope of the piecewise-linear function is altered. The unit generation cost within the interval $\left[p_{g}^{\kappa}, p_{g}^{\kappa+1}\right)$ is denoted with $v c_{g}^{\kappa}$. Similar to the start up costs, we assume that $v c_{g}^{\kappa_{g}^{\max }} \geq \ldots \geq v c_{g}^{1}, \forall g \in \mathcal{G}$, which suggests that the marginal costs increase as the outputs approach the generator capacities. Finally, the aggregate cost of generating $p_{g}^{\kappa}$ units of output is represented through the function $\hat{V}\left(p_{g}^{\kappa}\right)$. Following these definitions, the production amounts can be accounted by the variables $v_{g, t}$ with the following production-cost constraints:

$$
\begin{aligned}
v_{g, t} \geq v c_{g}^{\kappa}\left(p_{g, t}-p_{g}^{\kappa-1}\right)+\hat{V}_{g}\left(p_{g}^{\kappa-1}\right) \\
\quad \forall g \in \mathcal{G}, t \in \mathcal{T}, \text { and } \forall \kappa=\left\{1 \ldots \kappa_{g}^{\max }\right\} .
\end{aligned}
$$

Above, we assume that $p_{g}^{0}=0$ (and hence, $\hat{V}_{g}\left(p_{g}^{0}\right)=0$ ).

Along with others, the use of $p_{g, t}^{\prime}$ variables also eases the formulation of piecewise-linear production costs. Recall that the total production of a generator is now accounted by two terms, $p_{g, t}^{\prime}$ and $\underline{C}_{g} x_{g, t}$. As long as the generator is operational, the cost of producing the initial $\underline{C}_{g}$ units of energy is fixed and can be computed a-priori (herein denoted as $\hat{V}_{g}\left(\underline{C}_{g}\right)$ ). This cost can be associated with the $s_{g, t}$ and $\tilde{x}_{g, t}$ variables and directly accounted in the objective function. Therefore, the variables $v_{g, t}$ now provides the cost of producing an amount of energy in addition to $\underline{C}_{g}$. This cost is computed by the following production-cost constraints:

$$
\begin{array}{r}
v_{g, t} \geq v c_{g}^{\kappa}\left(p_{g, t}^{\prime}+\underline{C}_{g}-p_{g}^{\kappa-1}\right)+\hat{V}_{g}\left(p_{g}^{\kappa-1}\right)-\hat{V}_{g}\left(\underline{C}_{g}\right) \\
\forall g \in \mathcal{G}, t \in \mathcal{T}, \text { and } \forall \kappa=\left\{l_{g} \ldots \kappa_{g}^{\max }\right\} .
\end{array}
$$

Above, $l_{g}(\geq 1)$ is the value of the index $\kappa$ for which $p_{g}^{l_{g}-1}=$ $\underline{C}_{g}$. For values of $\kappa \in\left\{1 \ldots l_{g}-1\right\}$, constraints (14) are omitted because their right-hand side will be no larger than zero and will be trivially satisfied due to the nonnegativity of the variables.

\section{B. Benchmark formulations}

Using the inequalities presented in the previous section, we provide two complete UC formulations, which are primarily based on [13] and [21], respectively. The formulations are named after the initials of their corresponding authors.

$$
\begin{aligned}
& \text { OAV: } \min \sum_{t \in \mathcal{T}} \sum_{g \in \mathcal{G}}\left(f_{g, t}+v_{g, t}\right) \\
& \text { s.t. }(1 \mathrm{a}),(1 \mathrm{~b}),(2),(1 \mathrm{~d}),(3),(7),(9),(11),(13) \text {, } \\
& (\mathbf{x}, \mathbf{s}, \mathbf{z}) \in\{0,1\}^{3|\mathcal{G}||\mathcal{T}|},(\mathbf{p}, \overline{\mathbf{p}}, \mathbf{f}, \mathbf{v}) \geq \mathbf{0} \text {. } \\
& \text { MLR: } \min \sum_{t \in \mathcal{T}} \sum_{g \in \mathcal{G}}\left(\underline{C}_{g} x_{g, t}+v_{g, t}+\sum_{\tau \in \mathcal{I}_{g}} f c_{g}^{\tau} \delta_{g, t, \tau}\right) \\
& \text { s.t. (1a), 44, (5), 66, 77, 8), (12), 14, } \\
& (\mathbf{x}, \mathbf{s}, \mathbf{z}) \in\{0,1\}^{3|\mathcal{G}||\mathcal{T}|},\left(\mathbf{p}^{\prime}, \mathbf{r}, \boldsymbol{\delta}, \mathbf{v}\right) \geq \mathbf{0} \text {. }
\end{aligned}
$$

\section{The state-transition formulation}

In this section, we present the state-transition formulation (STF) for the UC problem. We develop this formulation through the use of the following state-transition variables:

$\tilde{x}_{g, t}: \quad 1$ if $g$ remains operational at time $t, 0$ otherwise.

$\tilde{s}_{g, t} / \tilde{z}_{g, t}: 1$ if $g$ is turned on / off at time $t, 0$ otherwise,

To see how these variables capture the transition of generator states, we provide an illustration in Fig. 1. In this figure, the on/off status of a generator is denoted with nodes and the feasible state transitions are represented with arcs. Notice that the variables for the decision remaining off has not been defined and the corresponding arc in Fig 1 is marked with a dashed line. This is because such a transition is completely determined by the values of the other state-transition variables. Indeed, exactly one state transition occurs at each time period, and the corresponding decision variable is set to 1 . If none of these variables are set to 1 , then the remaining off transition corresponding to the dashed line is said to occur. Following this discussion, it is easy to see that the inequality

$$
\tilde{s}_{g, t}+\tilde{x}_{g, t}+\tilde{z}_{g, t} \leq 1 \quad \forall g \in \mathcal{G}, t \in \mathcal{T} .
$$

is true for any feasible generator schedule formulated with state-transition variables.

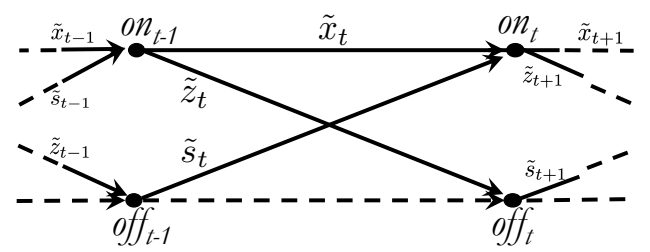

Fig. 1. Illustration of the state-transition variables.

A deeper look at Fig 1 reveals the network sub-structure embedded in the new formulation. In particular, we observe that a sequence of state transitions of a single generator can be de facto represented by a path on the graph comprising states as nodes and state transitions as arcs. Writing out the flow conservation constraints at the nodes of this graph, we immediately obtain the following state-transition constraints for the UC problem:

$$
\tilde{s}_{g, t-1}+\tilde{x}_{g, t-1}=\tilde{z}_{g, t}+\tilde{x}_{g, t} \quad \forall g \in \mathcal{G}, t \in \mathcal{T} .
$$


Constraints (16) are the flow conservation constraints at the nodes where the state of the generator is on. At the off nodes, the flow conservation constraints are the same, therefore omitted. This can be easily verified by assigning the expression $1-s_{g, t}-\tilde{x}_{g, t}-z_{g, t}$ to the dashed arc in Fig 1 . From our perspective, these constraints indicate that if a generator is operational at time $t-1$ then it must either be turned off or remain on at time $t$.

The linear mapping $\left(x_{g, t}\right) \rightarrow\left(\tilde{s}_{g, t}+\tilde{x}_{g, t}\right)$ allows us to translate any constraint derived for the benchmark formulations into the constraints of our formulation. Although the constraints differ in essence, it is easy to verify that (16) can also be derived from (1a) using the transformation described above.

In a UC problem, the above formulation of the state transitions can potentially lead to significant computational gains, especially when large numbers of state transitions are anticipated. Accordingly, for problems involving longer planning horizons and facing variable demand patterns, one should expect a better performance from this new formulation, compared to contenders that use $(\mathbf{x}, \mathbf{s}, \mathbf{z})$ variables. While other components of a UC problem can be formulated in alternative ways, the constraints presented in the rest of this section perform well in tandem, and some naturally define the facets of their corresponding polytopes.

a) Formulating production: In our formulation, we opt for the $p_{g, t}^{\prime}$ and $\bar{p}_{g, t}$ variables for formulating production amounts and reserve requirements. With the state-transition variables, the on/off status of a generator is given by the expression $\tilde{s}_{g, t}+\tilde{x}_{g, t}$. Accordingly, the production limits for each $g \in \mathcal{G}, t \in \mathcal{T}$ can be given as follows:

$$
\begin{array}{r}
\bar{p}_{g, t} \geq p_{g, t}^{\prime}+\underline{C}_{g}\left(\tilde{s}_{g, t}+\tilde{x}_{g, t}\right), \\
\bar{p}_{g, t} \leq \bar{C}_{g}\left(\tilde{s}_{g, t}+\tilde{x}_{g, t}\right)+\left(\underline{S}_{g}-\bar{C}_{g}\right) \tilde{z}_{g, t+1} .
\end{array}
$$

Constraints 17a ensure that the maximum possible generation amount at time $t\left(\bar{p}_{g, t}\right)$ is greater than the actual production amount at the same time period, and constraints $17 \mathrm{~b}$ bound $\bar{p}_{g, t}$ by the total capacity or the shut down limit of the generator. The minimum production constraints are redundant due to the use of the $p_{g, t}^{\prime}$ variables. As is the case in (2), the variable $\bar{p}_{g, t}$ must also respect the start up limits, but this restriction is omitted in (17) as it will be implied by the ramping constraints.

The demand constraints of the STF are similar to that of MLR given in (5):

$$
\sum_{g \in \mathcal{G}}\left(p_{g, t}^{\prime}+\underline{C}_{g}\left(\tilde{s}_{g, t}+\tilde{x}_{g, t}\right)\right) \geq d_{t} \quad \forall t \in \mathcal{T} .
$$

Finally, the reserve requirements are formulated without effort by adopting the constraints in (3).

b) Formulating operational and technological restrictions: We now formulate the minimum up/downtime and the ramping restrictions. We begin with the former, and translate the minimum up/downtime inequalities (7) into our formulation as presented below:

$$
\begin{aligned}
\sum_{i=t-U T_{g}+1}^{t-1} \tilde{s}_{g, i} \leq \tilde{x}_{g, t} & \forall g \in \mathcal{G}, t \in \mathcal{T} \\
\sum_{i=t-D T_{g}}^{t} \tilde{s}_{g, i} \leq 1-\tilde{x}_{g, t-D T_{g}} & \forall g \in \mathcal{G}, t \in \mathcal{T}
\end{aligned}
$$

Constraint $19 \mathrm{a}$ ensures that if the generator remains on, it could have been turned on at most once in the previous $U T_{g}-$ 1 time periods. If it does not remain on, then it could not have been turned on in these time periods due to minimum uptime restrictions. Similarly, constraint (19b) ensures that if the generator remains on, it cannot be restarted in the current time period or in the next $D T_{g}$ time periods due to minimum downtime restrictions. On the other hand, if it does not remain on, it can be turned on at most once in these time periods, due to minimum uptime and downtime restrictions.

To formulate the ramping restrictions, we take advantage of both the state-transition and the production variables, and provide the following ramp up constraints:

$$
\bar{p}_{g, t}-p_{g, t-1}^{\prime} \leq \bar{S}_{g} \tilde{s}_{g, t}+\left(\bar{R}_{g}+\underline{C}_{g}\right) \tilde{x}_{g, t} \quad \forall g \in \mathcal{G}, t \in \mathcal{T} .
$$

The coefficient of the remain-on variable is increased by $\underline{C}_{g}$ because the $p_{g, t-1}^{\prime}$ variable only accounts for the power generation beyond $\underline{C}_{g}$. When a generator is turned on in period $t$, it should have been idle in period $t-1$. Therefore $p_{g, t-1}^{\prime}=0$ and no increment for the $\tilde{s}_{g, t}$ coefficient is necessary. The ramp down constraints are formed in a similar manner and given below.

$$
p_{g, t-1}^{\prime}-p_{g, t}^{\prime} \leq\left(\underline{S}_{g}-\underline{C}_{g}\right) \tilde{z}_{g, t}+\underline{R}_{g} \tilde{x}_{g, t} \quad \forall g \in \mathcal{G}, t \in \mathcal{T} .
$$

Whenever the generator remains on, the above inequality limits the reduction in the power output such that the ramp down restrictions are obeyed. When the generator is turned off at time $t$, the inequality reduces to a tight upper bound on the $p_{g, t-1}^{\prime}$ variable. When the reserve requirements are ignored, it can be shown that 20 is equivalent to 10 .

c) Linearization of the objective function: For the linearization of the start up costs, we opted for the use of the $f_{g, t}$ variables. Observe that, when turned on, the start up cost of a generator will at least be its warm-start cost, i.e., the start up cost incurred when the generator has not cooled down since the previous operational state. Due to the minimum downtime restriction, this cost is at least $f c_{g}^{D T_{g}}$. Furthermore, it can be associated with the start up variable and accounted directly in the objective function using the additional term $f c_{g}^{D T_{g}} s_{g, t}$. Therefore, the variable $f_{g, t}$ will now represent the extra cost of turning on a generator which has been idle for some time that is longer than its minimum downtime $\left(D T_{g}\right)$. In view of this observation, variables $f_{g, t}$ must satisfy the following start up cost constraints:

$$
\begin{array}{r}
f_{g, t} \geq\left(f c_{g}^{\tau}-f c_{g}^{D T_{g}}\right)\left(\tilde{s}_{g, t}-\sum_{i=D T_{g}}^{\tau} \tilde{s}_{g, t-i}-\tilde{x}_{g, t-\tau}\right) \\
\forall g \in \mathcal{G}, t \in \mathcal{T}, \text { and } \tau \in \mathcal{I}_{g} \backslash\left\{1 \ldots D T_{g}\right\}
\end{array}
$$

We note that, in general, the number of constraints to formulate the piecewise-linear cost functions can make mixedinteger programs extremely large. As in MLR, the representation above mitigates this potential issue by eliminating up to $|\mathcal{T}| \times \sum_{g \in \mathcal{G}} D T_{g}$ constraints.

Finally, the production costs can be formulated by directly incorporating (14) into our formulation. Similar to MLR, the cost of minimum required production is determined by $\underline{C}_{g}\left(\tilde{s}_{g, t}+\tilde{x}_{g, t}\right)$, and appended to the objective function. 
d) The state-transition formulation: A summary of the new formulation is provided below:

$$
\begin{aligned}
& \text { STF: } \min \sum_{t \in \mathcal{T}} \sum_{g \in \mathcal{G}} f_{g, t}+v_{g, t}+\left(f c_{g}^{D T_{g}}+\underline{C}_{g}\right) s_{g, t}+\underline{C}_{g} \tilde{x}_{g, t} \\
& \text { s.t. 33, (16)- 21], (14), }(\tilde{\mathbf{x}}, \tilde{\mathbf{s}}, \tilde{\mathbf{z}}) \in\{0,1\}^{3|\mathcal{G}||\mathcal{T}|},(\mathbf{p}, \overline{\mathbf{p}}, \mathbf{f}, \mathbf{v}) \geq \mathbf{0}
\end{aligned}
$$

In STF, we do not include constraints 15 as they are implied by minimum downtime constraints (see Appendix A].

\section{COMPuTATIONAL EXPERIMENTS}

Our analysis will focus on two classes of instances. The first stems from the synthetic instances of [13], which are based on [23]. These are single-day instances, involving 24 time periods, and are characterized by increasing numbers of generators. They are known to be challenging for branch-and-bound algorithms, therefore commonly experimented in the literature. Using these instances, we generated two additional data sets. In the first, the numbers of generators are increased tenfold, and in the latter, the time horizon is extended to seven days. Instances with a longer time horizon are more challenging not simply because of the increased dimensions but also due to the daily trends and fluctuations in the demand. The second class of instances are based on the realistic instances obtained through the FERC. Further details can be found in [30]. For both classes of instances, details are given in Appendix B and the references therein.

For dynamic planning models it is well known that the planning horizon can have a significant impact on the decisions. The resulting decisions are often myopic, and this phenomenon is commonly referred to as the planning horizon effect. Such myopic choices can be remedied by choosing to solve longer-horizon models, and only implementing the dayahead plan. This approach is sometimes also referred to as a receding horizon approach (RHA). In case of the UC model, since a week can be considered as a regeneration point for demands (see [31], [32]), such an RHA is likely to avoid myopic choices. In keeping with this outlook, the choice of a 168 periods reflects the UC problem for the RHA.

In Table [1, we present statistics regarding the considered formulations. We observe that the number of binary variables is the same across all formulations. In terms of the numbers of variables, constraints, and nonzero coefficients, STF attains the minimum amounts, leading to compact descriptions of UC problems. We note that the week-long realistic instance contains a significantly larger set of generators compared to other week-long instances that we have experimented with.

All runs were performed on a single thread of a Dell Desktop PC with Intel ${ }^{\circledR}$ Core $^{\mathrm{TM}}$ i7-3770S CPU @ $3.10 \mathrm{GHz}$, 7.68 GB of RAM, and running Ubuntu Linux 12.04.3 LTS. The formulations were solved with CPLEX 12.5.1. The default parameters of CPLEX were preserved, but a time limit of 2 hours is imposed. For instances which could not be solved within this limit, we report the relative optimality gap based on the best available solution and lower bound. It is important to note that the benchmark formulations are true to the mathematical representations provided by the original authors. In making comparisons, certain choices -such as software parameters- are kept the same across all formulations,
TABLE I

FORMULATION STATISTICS (SYNTHETIC INSTANCES WITH $|\mathcal{T}|=24$ CONTAIN TEN TIMES MORE GENERATORS)

\begin{tabular}{cc|rrr}
\hline & \multicolumn{4}{c}{ Synthetic Instances (averaged) } \\
\hline & $|\mathcal{T}|$ & Binary / Total Vars. & Constraints & Nonzeros \\
\hline OAV & 24 & $77,076 / 179,845$ & 304,789 & $1,178,779$ \\
& 168 & $53,953 / 125,892$ & 214,676 & 868,319 \\
\hline STF & 24 & $77,076 / 179,845$ & 231,313 & $1,012,799$ \\
& 168 & $53,953 / 125,892$ & 163,243 & 748,272 \\
\hline MLR & 24 & $77,076 / 205,537$ & 257,125 & $1,048,143$ \\
& 168 & $53,953 / 143,876$ & 181,312 & 769,461 \\
\hline & \multicolumn{4}{|c}{ Realistic Instances } \\
\hline OAV & 24 & $67,608 / 157,753$ & 287,108 & $1,050,268$ \\
& 168 & $473,256 / 1,104,265$ & $2,016,548$ & $9,327,706$ \\
\hline STF & 24 & $67,608 / 157,753$ & 225,188 & 925,739 \\
& 168 & $473,256 / 1,104,265$ & $1,583,108$ & $8,416,361$ \\
\hline MLR & 24 & $67,608 / 180,289$ & 241,532 & 945,153 \\
& 168 & $473,256 / 1,262,017$ & $1,697,516$ & $8,519,871$ \\
\hline
\end{tabular}

and no tuning was performed to individually improve their performances.

In Table II] we report the solution times for the synthetic instances, along with the numbers of cuts and nodes generated by the solver. When accompanied with fast solution times, small numbers of cuts could indicate that the instances are amenable to producing integer solutions fast and with little need for tightening. Likewise, small numbers of branch-andbound nodes (or the lack thereof) imply that the nonconvexity in the instances can be easily tamed. We first make comparisons on the single-day instances. We observe that STF and MLR perform considerably faster than OAV, especially as the number of generators grow. In terms of solution times, there does not appear to be a significant contender among STF and MLR. However, it is promising to see that the total solution time for all instances is the smallest when STF is used, and the individual times never exceed a minute. Moreover, with STF, all of the instances were solved at the root node, with the minimum number for cutting planes. In contrast, we observe that multiple nodes were explored in a few instances using the benchmark formulations. When we consider week-long instances, we observe an important feature of our formulation. Recall from 8 II-C that the STF involves an implicit network sub-structure, and it has better potential in capturing the state transitions of the generators between consecutive time periods. Aligned with our expectations in $\$$ II-C we observe that the complexity introduced by a longer time horizon is best tamed with STF. The solution times are significantly better than the benchmark formulations for almost every instance. Furthermore, there are only two instances for which multiple branch-and-bound nodes have been explored. In contrast, the benchmark formulations needed to explore hundreds of nodes to optimize a significant portion of the instances. These results reveal the potential of our formulation to scale up to more realistic instances solved by the power industry.

We turn our attention to our realistic instances. We consider both a day-long and a week-long version of the instance 
TABLE II

PERFORMANCE MEASURES OF BRANCH-AND-BOUND FOR SYNTHETIC INSTANCES.

\begin{tabular}{|c|c|c|c|c|c|c|c|c|c|c|c|c|c|}
\hline \multirow{2}{*}{$\begin{array}{l}|\mathcal{T}|=24 \\
|\mathcal{G}|\end{array}$} & \multicolumn{2}{|l|}{ OAV } & \multicolumn{2}{|l|}{ STF } & \multicolumn{2}{|l|}{ MLR } & \multicolumn{3}{|c|}{$\||\mathcal{T}|=168$} & \multicolumn{2}{|l|}{ STF } & \multicolumn{2}{|l|}{ MLR } \\
\hline & Cuts/Nodes & Time & Cuts/Nodes & Time & Cuts/Nodes & Time & $|\mathcal{G}|$ & Cuts/Nodes & Time & Cuts/Nodes & Time & Cuts/Nodes & Time \\
\hline 280 & $-1-$ & 2.5 & $-1-$ & 1.9 & $-1-$ & 1.1 & 28 & $428 /-$ & 3.3 & $12 /-$ & 3.4 & $252 / 170$ & 5.8 \\
\hline 350 & $506 /-$ & 5.2 & $-1-$ & 2.7 & $-1-$ & 1.6 & 35 & 914 / - & 6.4 & $-1-$ & 2.1 & $865 /-$ & 4.4 \\
\hline 450 & $3,867 /$ - & 34.4 & $218 /-$ & 5.0 & $1,019 / 100$ & 12.4 & 45 & $3,016 / 417$ & 34.3 & $158 /-$ & 4.7 & 984 / 413 & 12.5 \\
\hline 490 & $6,334 /-$ & 29.8 & $-1-$ & 4.7 & $63 /-$ & 5.9 & 49 & $2,981 / 210$ & 25.1 & $92 / 75$ & 8.7 & $517 / 506$ & 21.3 \\
\hline 500 & $9,520 /-$ & 83.5 & $863 /-$ & 8.5 & $2,142 / 80$ & 24.7 & 50 & $3,191 / 529$ & 61.2 & $216 /-$ & 6.0 & $837 / 806$ & 23.1 \\
\hline 520 & $6,043 /-$ & 26.8 & $-1-$ & 4.6 & $1 /-$ & 3.8 & 52 & $2,099 /$ - & 14.5 & $100 /-$ & 4.7 & $469 /$ - & 6.4 \\
\hline 540 & 7,299 / - & 36.0 & $-1-$ & 4.6 & $12 /-$ & 4.4 & 54 & $2,470 /-$ & 14.7 & $88 / 63$ & 8.2 & $373 / 739$ & 21.9 \\
\hline 1320 & $2,676 /-$ & 35.9 & $-1-$ & 16.1 & $-1-$ & 9.8 & 132 & $1,206 /-$ & 32.9 & $-1-$ & 11.9 & $2,159 /-$ & 18.8 \\
\hline 1560 & $12,059 /$ - & 187.2 & $-1-$ & 23.4 & $1 / 10$ & 33.4 & 156 & $2,781 /-$ & 60.7 & $51 /-$ & 23.5 & $285 / 200$ & 39.6 \\
\hline 1560 & $12,737 /$ - & 111.6 & $-1-$ & 21.6 & $-1-$ & 19.4 & 156 & $3,413 /-$ & 48.3 & $-1-$ & 15.7 & $1,994 / 342$ & 52.0 \\
\hline 1650 & $17,249 /$ - & 133.1 & $-1-$ & 28.6 & $-1-$ & 23.9 & 165 & $6,564 /-$ & 102.8 & $-1-$ & 21.3 & $3,306 / 531$ & 89.6 \\
\hline 1670 & $19,588 /$ - & 230.5 & $-1-$ & 29.6 & $-1-$ & 27.0 & 167 & $8,292 /-$ & 111.1 & $243 /-$ & 22.1 & $638 / 1,880$ & 432.2 \\
\hline Total: & $03,787 / 774$ & $3,854.3$ & $2,503 /-$ & 367.8 & $7,937 / 685$ & 629.2 & & $82,769 / 1,296$ & $1,231.8$ & $2,591 / 138$ & 286.3 & $26,763 / 7,093$ & $1,098.8$ \\
\hline
\end{tabular}

made accessible by [30]. The analysis of these instances will provide better intuition on how the new and the benchmark formulations perform in the problems solved in the energy industry. Comparing Table [II with Table II] we observe that the differences in the computational performance of the new model and the benchmark formulations are more pronounced. For instance, we observe that OAV hits the time limit on the week-long problem, whereas MLR spends more than 20 minutes to obtain an optimal solution. In comparison, the new model can provide an optimal solution within 12 minutes, achieving a $45 \%$ reduction. We conclude our analysis by discussing the percentage of fractional variables in Table III. A small number of fractional variables in the continuous relaxation of the problem could serve as an indication of the tightness of a formulation. Observe that the new formulation provides far larger numbers of integer variables in the linearprogramming (LP) relaxations of the problems, confirming the effectiveness of the formulation. The objective value of the LP-relaxation of STF can be $1.4 \%$ and $0.2 \%$ better than that of OAV and MLR, respectively. More importantly, in the root relaxation (i.e., the starting LP relaxation created within CPLEX), the percentage of fractional variables shrink even further. Indeed, in the week-long instance, $99.82 \%$ of the integer variables are observed to be integral, leading to an almost-integer (lower-bounding) solution.

In order to further assess the behavior of the formulations, we have experimented with modified versions of the original synthetic and realistic instances. In particular, the hourly demand and reserve data are multiplied with $(1+\alpha)$, where $\alpha$ is chosen to mimic realistic changes in demand (see [33]). Generator capacities are perturbed with the same coefficient for synthetic instances, however, kept the same for realistic instances, in order to preserve their authenticity. Table IV gives a summary of our analysis. Examining the results for
TABLE III

PERFORMANCE MEASURES OF BRANCH-AND-BOUND AND THE $\%$ OF FRACTIONAL VARIABLES $(|\mathcal{G}|=939$, RELATIVE OPTIMALITY GAP IS REPORTED -IN BRACKETS- WHEN THE TIME LIMIT IS HIT)

\begin{tabular}{cccrrrr}
\hline & & \multicolumn{2}{c}{ Branch-and-Bound } & & Frac. Vars. (\%) \\
& $|\mathcal{T}|$ & Cuts/Nodes & Time & & LP/Root Relaxation \\
\hline OAV & 24 & $4,499 /-$ & 264.1 & & $2.19 / 0.15$ \\
& 168 & $15,605 /-$ & {$[0.5 \%]$} & & $1.54 / 0.37$ \\
\hline \multirow{2}{*}{ STF } & 24 & $478 / 10$ & 53.6 & & $1.09 / 0.13$ \\
& 168 & $1,940 /-$ & 679.8 & & $0.73 / 0.18$ \\
\hline \multirow{2}{*}{ MLR } & 24 & $2,914 /-$ & 66.9 & & $2.39 / 0.20$ \\
& 168 & $11,566 /-$ & $1,243.7$ & & $1.76 / 0.29$ \\
\hline
\end{tabular}

synthetic instances, we observe that STF is slightly more favored than MLR in the day-long problems, and performs significantly better for the week-long instances. A similar outcome is also observed for the realistic instances, although, for these instances, the performance delivered by STF is consistently better. Notice that the computational gains observed for the week-long realistic instances are much larger than the total gains observed for the 20 week-long synthetic instances, indicating the potential of STF for instances with more realistic and diverse problem parameters (e.g., distinct generator capacities, ramping rates, or variable-cost functions with many more pieces).

In our final analysis, we demonstrate the value of considering longer planning horizons in UC problems. Single-day problems are not designed to capture the day to day dynamics of a continuously-evolving demand pattern, and may lead to suboptimal decisions at later periods of the planning horizon. We show this on the realistic instances, by solving 7 sequential day-long UC problems, and their week-long counterpart. Fig. 2 illustrates the optimal production patterns of two generators 
TABLE IV

SOLUTION TIMES FOR MODIFIED INSTANCES $(|\mathcal{G}|$ IS 10 -FOLD WHEN $|\mathcal{T}|=24$ FOR SYNTHETIC INSTANCES, AND $|\mathcal{G}|=939$ FOR REALISTIC INSTANCES).

\begin{tabular}{|c|c|c|c|c|c|c|c|}
\hline & & \multicolumn{6}{|c|}{ Synthetic Instances (summed over 20 instances) } \\
\hline & $|\mathcal{T}|$ & $\alpha=-5 \%$ & $-2.5 \%$ & $-1 \%$ & $1 \%$ & $2.5 \%$ & $5 \%$ \\
\hline \multirow[t]{2}{*}{ OAV } & 24 & 4407.2 & 4662.3 & 4363.8 & 3699.6 & 4177.9 & 5343.6 \\
\hline & 168 & 1340.2 & 1154.8 & 1210.1 & 1538.2 & 1484.0 & 1478.8 \\
\hline \multirow[t]{2}{*}{ STF } & 24 & 397.0 & 367.4 & 371.8 & 414.6 & 491.7 & 597.5 \\
\hline & 168 & 301.2 & 287.3 & 300.6 & 319.8 & 371.7 & 348.2 \\
\hline \multirow[t]{4}{*}{ MLR } & 24 & 394.3 & 441.2 & 367.1 & 451.1 & 546.0 & 579.3 \\
\hline & 168 & 730.4 & 757.3 & 1111.0 & 1293.1 & 916.2 & 904.0 \\
\hline & & \multicolumn{6}{|c|}{ Realistic Instances } \\
\hline & $|\mathcal{T}|$ & $\alpha=\mathbf{- 5 \%}$ & $-2.5 \%$ & $-1 \%$ & $1 \%$ & $2.5 \%$ & $5 \%$ \\
\hline \multirow[t]{2}{*}{ OAV } & 24 & 280.3 & 328.6 & 233.8 & 294.5 & 167.3 & 254.7 \\
\hline & 168 & {$[0.5 \%]$} & {$[0.7 \%]$} & {$[0.5 \%]$} & {$[0.8 \%]$} & {$[0.4 \%]$} & {$[0.4 \%]$} \\
\hline \multirow[t]{2}{*}{ STF } & 24 & 30.4 & 33.8 & 35.8 & 36.0 & 35.8 & 33.9 \\
\hline & 168 & 835.7 & 523.2 & 761.8 & 712.5 & 497.2 & 658.0 \\
\hline \multirow[t]{2}{*}{ MLR } & 24 & 58.9 & 47.7 & 43.5 & 49.5 & 37.7 & 38.4 \\
\hline & 168 & 1417.7 & 1847.9 & 1329.7 & 1336.2 & 1362.2 & 1338.1 \\
\hline
\end{tabular}

under the commitment decisions of these runs. The same pattern appears for most nonbase-load generators. The daylong problems recommend turning these generators off at the end of each day. In contrast, the week-long problem acts with better foresight and recommends less-frequent state transitions, leading to more stable commitment schedules. More importantly, the week-long problem reports $1.8 \%$ lower operational costs.

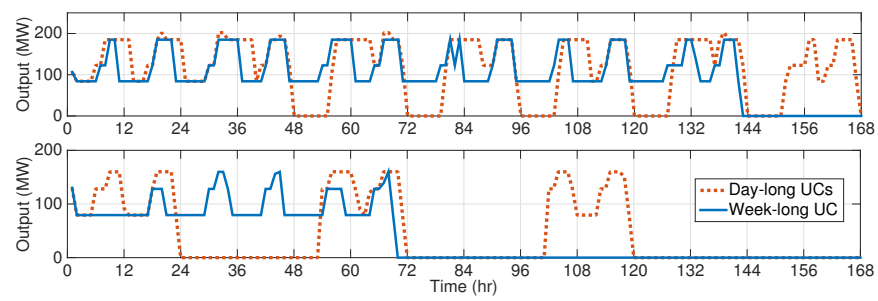

Fig. 2. Production patterns of two generators based on the commitment decisions of 7 day-long UC problems, and a week-long problem.

\section{CONCLUSION}

In this study, we developed the state-transition formulation for the UC problem. Our formulation replaces the long-established state variables of the UC formulations with state-transition variables. We compare the performance of the new formulation with two benchmark formulations. We observe that the state-transition formulation performs the best especially for long-horizon instances. The new formulation naturally includes valid inequalities that have been used to strengthen alternative formulations. The induced network substructure of the formulation allows the effect of these valid inequalities to propagate throughout the planning horizon.

\section{APPENDIX A}

INSIGHTS ON THE POLYHEDRAL STRUCTURE

This appendix summarizes the polyhedral features of our formulation and draws connections with other formulations from the literature. Our discussion will focus on modeling components that involve a single generator, therefore the generator indices are neglected for notational ease. We begin by showing the redundancy of (15) in STF.

Proposition 1. Constraints (15) are implied by the minimum downtime constraints (19b) and the state-transition constraints (16).

Proof. Consider constraint (19b) for time $t+D T-1$,

$$
\begin{aligned}
1 & \left.\geq \tilde{x}_{t-1}+\sum_{i=t-1}^{t+D T-1} \tilde{s}_{i}=\tilde{x}_{t-1}+\tilde{s}_{t-1}+\sum_{i=t}^{t+D T-1} \tilde{s}_{i} \quad \text { [by [16] }\right] \\
& =\tilde{z}_{t}+\tilde{x}_{t}+\tilde{s}_{t}+\sum_{i=t+1}^{t+D T-1} \tilde{s}_{i} \geq \tilde{z}_{t}+\tilde{x}_{t}+\tilde{s}_{t} .
\end{aligned}
$$

Remark 1. If $D T=1$, constraints (15) become equivalent to the minimum downtime constraints.

The following definitions and facts will be useful in the remainder of this appendix. We use $\operatorname{dim}(\mathcal{P})$ to denote the dimension of a polyhedron $\mathcal{P}$. A facet $\mathcal{F}$ of $\mathcal{P}$ has $\operatorname{dim}(\mathcal{F})=$ $\operatorname{dim}(\mathcal{P})-1$. Accordingly, to prove that a constraint $\pi^{\top} x \leq \pi_{0}$ defines a facet of $\mathcal{P}$, it is sufficient to identify $\operatorname{dim}(\mathcal{P})$ affinelyindependent points $\left(x^{i}\right)$ which satisfy $\pi^{\boldsymbol{\top}} x^{i}=\pi_{0}, \forall i=$ $1 \ldots \operatorname{dim}(\mathcal{P})$. A facet-defining inequality will dominate all other constraints, therefore sought in most polyhedral analyses. For proofs of this type appealing to affine independence for facet-defining inequalities, the reader may refer to [34].

We show that the use of the state-transition decision variables does not affect the polyhedral properties of the original minimum up/downtime constraints [19] of [11], proposed for formulations with $(\mathbf{x}, \mathbf{s}, \mathbf{z})$ variables. Consider the minimum up/downtime polytope of the STF:

$$
\begin{array}{rr}
\mathcal{M}=\{\tilde{\mathbf{s}}, \tilde{\mathbf{x}}, \tilde{\mathbf{z}} \in\{0,1\}|\mathcal{T}|: & \\
\sum_{i=t-U T+1}^{t-1} \quad \tilde{s}_{i} \leq \tilde{x}_{t} & \forall t \in\{U T \ldots|\mathcal{T}|\}, \\
\sum_{i=t-D T}^{t} \quad \tilde{s}_{i} \leq 1-\tilde{x}_{t-D T} & \forall t \in\{D T+1 \ldots|\mathcal{T}|\}, \\
\tilde{s}_{t-1}+\tilde{x}_{t-1}=\tilde{z}_{t}+\tilde{x}_{t} & \forall t \in\{2 \ldots|\mathcal{T}|\} .\}
\end{array}
$$

In the definition above, the history of the generators is neglected by removing the corresponding turn on/off constraints defined for periods $\{1 \ldots U T-1\}$, and $\{1 \ldots D T\}$, respectively.

Let $\operatorname{conv}(\mathcal{P})$ to denote the convex hull of polytope $\mathcal{P}$.

Proposition 2. The minimum up/downtime constraints (19) define the facets of the polytope $\operatorname{conv}(\mathcal{M})$.

Proof. Observing the linear independence of equations in (16), $\operatorname{dim}(\operatorname{conv}(\mathcal{M}))$ can at most be $2 \times|\mathcal{T}|+1$. This is indeed true because the following $2 \times|\mathcal{T}|+2$ integer solutions of $\mathcal{M}$ are affinely-independent. To see this, note that by ordering the solution in a matrix and placing first the $\tilde{\mathbf{x}}$ variables and then the $\tilde{\mathbf{s}}$ and the $\tilde{\mathbf{z}}$ variables, we obtain a lower-triangular submatrix. 
- Consider a set of $|\mathcal{T}|$ solutions $(\tilde{\mathbf{s}}, \tilde{\mathbf{x}}, \tilde{\mathbf{z}})_{i}$, where $i \in$ $\{1 \ldots|\mathcal{T}|\}$. This solution has the following structure. The components of the vector $\mathbf{s}$ (resp. $\mathbf{z}$ ) are set to zero with the only exception of the $i^{t h}$ component (resp. $(i+U T+1)^{t h}$ or $|\mathcal{T}|$, whichever is smaller). This element is set to 1 . The components of $\tilde{\mathbf{x}}$ are set as

$$
\tilde{x}_{k}=\left\{\begin{array}{lr}
1, & i+1 \leq k \leq \min \{i+U T,|\mathcal{T}|\} \\
0, & \text { otherwise. }
\end{array}\right.
$$

- $|\mathcal{T}|$ additional solutions $(\tilde{\mathbf{s}}, \tilde{\mathbf{x}}, \tilde{\mathbf{z}})_{j}, j \in\{1 \ldots|\mathcal{T}|\}$, which belong to a set $J$, are obtained by setting the $\tilde{\mathbf{s}}$ and $\tilde{\mathbf{z}}$ vectors to null, while the vector $\tilde{\mathbf{x}}$ has the following components:

$$
\tilde{x}_{k}=\left\{\begin{array}{lr}
1, & k \leq j \\
0, & \text { otherwise. }
\end{array}\right.
$$

- The last two solutions are $(\tilde{\mathbf{s}}, \tilde{\mathbf{x}}, \tilde{\mathbf{z}})=(\mathbf{0}, \mathbf{0},(1,0, \ldots 0))$ and the null solution.

Given the dimension of $\operatorname{conv}(\mathcal{M})$, any constraint $(19 \mathrm{a})$ is a facet if it has dimension $2 \times|\mathcal{T}|$ (i.e., if there are $2 \times|\mathcal{T}|+1$ affinely independent solutions which satisfy the inequality as equality). For some $t \in\{U T \ldots|\mathcal{T}|\}$, consider the set

$$
\mathcal{U}_{t}=\left\{\tilde{\mathbf{s}}, \tilde{\mathbf{x}}, \tilde{\mathbf{z}} \in\{0,1\}^{|\mathcal{T}|}: \sum_{i=t-U T+1}^{t-1} \tilde{s}_{i}=\tilde{x}_{t}\right\} .
$$

All the solutions listed above belong to the set $\mathcal{U}_{t}$, with the exception of $|\mathcal{T}|-(t+1)$ solutions of set $J$. In these solutions, the generator is turned off at time $t+1$ or later (i.e., all solutions $(\tilde{\mathbf{s}}, \tilde{\mathbf{x}}, \tilde{\mathbf{z}})_{j}$ where $j>t$ ). For each of these solutions, turn the generator on at time $t-U T+1$ by setting the $(t-U T+1)^{t h}$ component of the vector $\mathbf{s}$ to 1 , and setting the first $t-U T+1$ components of the vector $\tilde{\mathbf{x}}$ to 0. $2 \times|\mathcal{T}|+1$ affinely independent solutions have thus been generated, proving that $\mathcal{U}_{t}$ is a facet of $\operatorname{conv}(\mathcal{M})$.

The proof for minimum downtime constraints $19 \mathrm{~b}$ is analogous and can be obtained with the same arguments made for constraints (19a).

Next, we show the validity of the ramping inequalities 20], and demonstrate their relation to their counterparts in [13].

Proposition 3. For each $t \in \mathcal{T}$, the ramp-up inequality 20a is valid and dominates constraint

$$
\bar{p}_{t}-p_{t-1} \leq \bar{S} \tilde{s}_{t}+\bar{R} \tilde{x}_{t},
$$

which is equivalent to 9a when the state-transition variables are used.

Proof. It is straightforward to verify the validity of constraints (20a). The inequality limits the increase in production by $\bar{S}$ if the generator has just been turned on (in this case $p_{t-1}^{\prime}=0$ ), or by $(\bar{R}+\underline{C})$ if it remains on. As also pointed out in II-A the ramp-up inequality $(\overline{\mathrm{P} 1 \mathrm{a}})$ is inactive if the generator has just been turned off; but it can be strengthened by lifting it to the space of $\tilde{z}_{t}$ variables.

$$
\bar{p}_{t}-\left[p_{t-1}^{\prime}+\underline{C}\left(\tilde{s}_{t-1}+\tilde{x}_{t-1}\right)\right] \leq \bar{S} \tilde{s}_{t}+\bar{R} \tilde{x}_{t}-\underline{C} \tilde{z}_{t} .
$$

The term within brackets is $p_{t-1}$. To verify that $(\overline{\mathrm{P} 1 \mathrm{~b}})$ is valid, it is sufficient to consider the case where $\tilde{z}_{t}=1$ (the case $\tilde{z}_{t}=$ 0 is trivial). As the generator is not operational at time period $t$, $\bar{p}_{t}$ is 0 and the inequality reduces to $p_{t-1}^{\prime}+\underline{C}\left(\tilde{s}_{t-1}+\tilde{x}_{t-1}\right)=$ $p_{t-1} \geq \underline{C}$, which is the minimum production requirement. Therefore $(\mathrm{P} 1 \mathrm{~b})$ is valid and dominates $(\mathrm{P} 1 \mathrm{a})$, which is easy to see as the left-hand side of (P1a) assumes no smaller value than the left-hand side of $(\overline{P 1 b})$. Constraints $(20 \mathrm{a})$ can then be obtained from (P1b) using (16):

$$
\begin{aligned}
\bar{p}_{t}-p_{t-1}^{\prime} & \leq \bar{S} \tilde{s}_{t}+\bar{R} \tilde{x}_{t}-\underline{C} \tilde{z}_{t}+\underline{C}\left(\tilde{s}_{t-1}+\tilde{x}_{t-1}\right) \\
& =\bar{S} \tilde{s}_{t}+\bar{R} \tilde{x}_{t}-\underline{C} \tilde{z}_{t}+\underline{C}\left(\tilde{z}_{t}+\tilde{x}_{t}\right) \\
& =\bar{S} \tilde{s}_{t}+(\bar{R}+\underline{C}) \tilde{x}_{t} .
\end{aligned}
$$

The analysis for the ramp down constraints is provided next.

Proposition 4. For each $t \in \mathcal{T}$, the ramp-down inequality 20b is valid and dominates the seed inequality $p_{t-1}-p_{t} \leq$ $\underline{S} \tilde{z}_{t}+\underline{R} \tilde{x}_{t}$, which is equivalent to (9b) when the state-transition variables are are used.

Proof. Consider the seed inequality:

$\left[p_{t-1}^{\prime}+\underline{C}\left(\tilde{s}_{t-1}+\tilde{x}_{t-1}\right)\right]-\left[p_{t}^{\prime}+\underline{C}\left(\tilde{s}_{t}+\tilde{x}_{t}\right)\right] \leq \underline{S} \tilde{z}_{t}+\underline{R} \tilde{x}_{t}$,

which can be strengthened by a lifting to the space of $\tilde{s}_{t}$,

$\left[p_{t-1}^{\prime}+\underline{C}\left(\tilde{s}_{t-1}+\tilde{x}_{t-1}\right)\right]-\left[p_{t}^{\prime}+\underline{C}\left(\tilde{s}_{t}+\tilde{x}_{t}\right)\right] \leq \underline{S} \tilde{z}_{t}+\underline{R} \tilde{x}_{t}-\underline{C} \tilde{s}_{t}$.

When $\tilde{s}_{t}=1$, the power output at time $t-1$ must be 0 , and the first term in brackets disappears. Since $\tilde{x}_{t}=\tilde{z}_{t}=0$, the inequality simply reduces to $p_{t}^{\prime} \geq 0$, which confirms its validity. By simple algebra, we obtain

$$
\begin{aligned}
p_{t-1}^{\prime}-p_{t}^{\prime} & \leq \underline{S} \tilde{z}_{t}+(\underline{R}+\underline{C}) \tilde{x}_{t}-\underline{C}\left(\tilde{s}_{t-1}+\tilde{x}_{t-1}\right) \\
& =\underline{S} \tilde{z}_{t}+(\underline{R}+\underline{C}) \tilde{x}_{t}-\underline{C}\left(\tilde{z}_{t}+\tilde{x}_{t}\right) \\
& =(\underline{S}-\underline{C}) \tilde{z}_{t}+\underline{R} \tilde{x}_{t}
\end{aligned}
$$

where ( $\mathrm{P} 2)$ is achieved using (16).

Using the relations (1a) and [16), it is trivial to verify the following remark.

Remark 2. When the reserve requirements are neglected, the ramping inequalities (20a) and (20b) of the new formulation are equivalent to the facet-defining two-period ramping inequalities of the base formulation, proposed by [14], i.e., constraints (10a) and (10b). In contrast, the ramping constraints in MLR are such that the state variables do not explicitly guide the ramping rates of generators.

For our final analysis, we introduce the two-period ramping polytope in our formulation, under the assumption that $U T>$ 1. For clarity of exposition, we first give the generation and ramping constraints for a given $t$ as follows:

$$
\begin{aligned}
& \bar{p}_{t} \geq p_{t}^{\prime}+\underline{C}\left(\tilde{s}_{t}+\tilde{x}_{t}\right) \\
& \bar{p}_{t} \leq \bar{S} \tilde{s}_{t}+\bar{C} \tilde{x}_{t}+(\underline{S}-\bar{C}) \tilde{z}_{t+1} \\
& p_{t-1}^{\prime}-p_{t}^{\prime} \leq(\underline{S}-\underline{C}) \tilde{z}_{t}+\underline{R} \tilde{x}_{t} \\
& \bar{p}_{t}-p_{t-1}^{\prime} \leq \bar{S} \tilde{s}_{t}+(\bar{R}+\underline{C}) \tilde{x}_{t}+(\underline{S}-\bar{R}-\underline{C}) \tilde{z}_{t+1} .
\end{aligned}
$$

It is easy to see that 23b is a tightened version of 17b), whereas 23d is the lifted version of the ramp up inequality (20a). Our computations do not consider (23b) and (23d) in order to simplify the exposition, and avoid detracting from the paper's main messages. It should also be noted that 23d is 
equivalent to the valid inequality (23) of [13], when written in terms of the state-transition and $p_{t-1}^{\prime}$ variables. We give the two-period ramping polytope below.

$$
\begin{aligned}
& R_{t}^{2}=\left\{\left(\bar{p}_{t}, p_{t-1}^{\prime}, p_{t}^{\prime}, \tilde{s}_{t}, \tilde{x}_{t}, \tilde{z}_{t} \tilde{z}_{t+1}\right) \in \Re_{+}^{3} \times\{0,1\}^{4}:\right. \\
& \text { 23a } \left., \text { 23b }, \text { 23c }, \text { 23d }, \tilde{s}_{t}+\tilde{x}_{t}+\tilde{z}_{t} \leq 1, \tilde{z}_{t+1} \leq \tilde{x}_{t}\right\}
\end{aligned}
$$

With respect to the two-period ramping polytope defined in [14], the above definition has some differences. For instance, we consider both the ramp up and ramp down constraints together, which were treated separately in [14]. Furthermore, our polytope is defined in the space of $\bar{p}_{t}, p_{t}^{\prime}, p_{t-1}^{\prime}, \tilde{s}_{t}, \tilde{x}_{t}, \tilde{z}_{t}$ and $\tilde{z}_{t+1}$ variables, and considers the reserve requirements.

Proposition 5. Assuming $\bar{C}>\bar{R}+\underline{R} \geq 2 \times \underline{R}$ and the generation limit constraints, (23a) and (23b), and the ramping down inequality (23c) and (23d) are facets of $\operatorname{conv}\left(R_{t}^{2}\right)$.

Proof. The dimension of $\operatorname{conv}\left(R_{t}^{2}\right)$ can at most be 7 . In Table V. we present feasible solutions for this polytope. The first 8 solutions are affinely-independent, thus proving the full dimensionality of $\operatorname{conv}\left(R_{t}^{2}\right)$.

TABLE V

\begin{tabular}{|c|c|c|c|c|c|c|c|c|}
\hline \# & $\bar{p}_{t}$ & $p_{t-1}^{\prime}$ & $p_{t}^{\prime}$ & $\tilde{s}_{t}$ & $\tilde{x}_{t}$ & $\tilde{z}_{t}$ & $\tilde{z}_{t+1}$ & Inactive Cons \\
\hline 1 & 0 & 0 & 0 & 0 & 0 & 0 & 0 & \\
\hline 2 & 0 & 0 & 0 & 0 & 0 & 1 & 0 & $23 c$ \\
\hline 3 & 0 & $\underline{S}-\underline{C}$ & 0 & 0 & 0 & 1 & 0 & $23 \mathrm{~d}$ \\
\hline 4 & $\bar{S}$ & 0 & 0 & 1 & 0 & 0 & 0 & $23 \mathrm{a}$ \\
\hline 5 & $\bar{S}$ & 0 & $\bar{S}-\underline{C}$ & 1 & 0 & 0 & 0 & $23 c$ \\
\hline 6 & $\bar{C}$ & $\bar{C}$ & $\bar{C}-\underline{R}$ & 0 & 1 & 0 & 0 & 23a; $23 \mathrm{~d}$ \\
\hline 7 & $\underline{R}+\underline{C}$ & $2 \underline{R}$ & $\underline{R}$ & 0 & 1 & 0 & 0 & $23 \mathrm{~b} ; 23 \mathrm{~d}$ \\
\hline 8 & $\underline{S}$ & $\underline{S}+\underline{R}-\underline{C}$ & $\underline{S}-\underline{C}$ & 0 & 1 & 0 & 1 & $23 \mathrm{~d}$ \\
\hline 9 & $\bar{R}+\underline{C}$ & $\underline{R}$ & 0 & 0 & 1 & 0 & 0 & \\
\hline
\end{tabular}

SET OF FEASIBLE SOLUTIONS FOR THE TWO-PERIOD RAMPING POLYTOPE.

Using the solutions displayed in Table V, it is also easy to verify that constraints (23a), 23b), 23c and (23d) are facetdefining for $\operatorname{conv}\left(R_{t}^{2}\right)$. In the last column of Table $\mathrm{V}$, we report the constraints which are not active in the corresponding solution. To prove that each of the constraints are facetdefining, we modify the coefficients of the solutions in Table $\mathrm{V}$ as follows.

- Constraint 23a): change the coefficient of $\bar{p}_{t}$ from $\bar{S}$ to $C$ in Solution 4.

- Constraint (23b): seven solutions out of the eight already satisfy the constraint as equality.

- Constraint (23c): substitute Solution 5 with Solution 9.

- Constraint (23d): change the coefficients of $p_{t-1}^{\prime}$ from $\bar{C}$ to $\bar{C}-\bar{R}-\underline{C}$, from $2 \underline{R}$ to 0 , and from $\underline{S}+\underline{R}-\underline{C}$ to $\underline{S}-\bar{R}-\underline{C}$ in Solutions 6,7 and 8 respectively.

For each of the constraints, we have provided six affinely independent feasible solutions which satisfy all constraints as equality, thus proving the proposition.

Remark 3. Observe that for every $t \in \mathcal{T}$, the inequality (15) is also facet-defining for $\operatorname{conv}\left(R_{t}^{2}\right)$.

\section{APPENDIX B \\ DETAILS ON DATA GENERATION}

\section{A. Synthetic Instances}

The instances studied in [13] consider a time horizon of 24 hours, where the hourly demands are given as percentages of the total generation capacity of the system, and the hourly reserve amounts are set to $3 \%$ of the resulting demand. To increase the number of generators, we duplicated all generators tenfold, as in [21]. To extend the time horizon, we utilized the demand pattern in our realistic instance (see Appendix B-B. For hour $h \in\{1 \ldots 24\}$ and day $d \geq 2$, we compute $d_{h, d} / d_{h, 1}$, where $d_{h, d}$ is the corresponding demand in the realistic instance. These ratios are multiplied with the demand percentages given in [13], and rounded to the nearest integer (see Table VI).

TABLE VI

HOURLY DEMANDS, GIVEN AS PERCENTAGES OF THE TOTAL GENERATION

\begin{tabular}{|c|c|c|c|c|c|c|c|c|c|c|c|c|c|c|c|}
\hline \multirow[b]{2}{*}{ Hrs } & \multicolumn{7}{|c|}{ Days } & & \multicolumn{7}{|c|}{ Days } \\
\hline & 1 & 2 & 3 & 4 & 5 & 6 & 7 & Hrs & 1 & 2 & 3 & 4 & 5 & 6 & $\overline{7}$ \\
\hline 1 & 71 & 68 & 66 & 64 & 63 & 63 & 63 & 13 & 82 & 87 & 87 & 85 & 83 & 87 & 83 \\
\hline 2 & 65 & 63 & 60 & 58 & 58 & 57 & 58 & 14 & 80 & 85 & 86 & 84 & 82 & 87 & 82 \\
\hline 3 & 62 & 60 & 58 & 55 & 55 & 55 & 55 & 15 & 79 & 84 & 86 & 84 & 82 & 87 & 82 \\
\hline 4 & 60 & 59 & 56 & 54 & 54 & 53 & 52 & 16 & 79 & 84 & 87 & 84 & 82 & 88 & 82 \\
\hline 5 & 58 & 58 & 55 & 52 & 53 & 52 & 50 & 17 & 83 & 88 & 91 & 88 & 86 & 92 & 85 \\
\hline 6 & 58 & 60 & 57 & 54 & 56 & 54 & 50 & 18 & 91 & 95 & 97 & 94 & 92 & 97 & 91 \\
\hline 7 & 60 & 66 & 63 & 59 & 62 & 59 & 52 & 19 & 90 & 93 & 93 & 91 & 89 & 92 & 89 \\
\hline 8 & 64 & 71 & 68 & 65 & 67 & 64 & 56 & 20 & 88 & 90 & 89 & 88 & 87 & 88 & 86 \\
\hline 9 & 73 & 80 & 76 & 74 & 75 & 73 & 65 & 21 & 85 & 87 & 85 & 84 & 84 & 83 & 82 \\
\hline 10 & 80 & 86 & 83 & 81 & 81 & 81 & 75 & 22 & 84 & 85 & 83 & 82 & 82 & 81 & 81 \\
\hline 11 & 82 & 87 & 86 & 84 & 83 & 84 & 79 & 23 & 79 & 79 & 77 & 76 & 76 & 76 & 77 \\
\hline 12 & 83 & 88 & 87 & 85 & 84 & 87 & 83 & 24 & 74 & 72 & 71 & 70 & 70 & 70 & 72 \\
\hline
\end{tabular}
CAPACITY OF THE SYSTEM $(\%)$.

\section{B. Realistic Instances}

The realistic instances of \$III are based on the winter test problem, made available by FERC ${ }^{1}$ and documented in [30]. Here, we only list our assumptions. We set $\bar{C}_{g}$ to the seasonal capabilities of the generators, and let $\bar{S}_{g}=$ (0.7) $\bar{R}_{g}, \underline{S}_{g}=(0.7) \underline{R}_{g} . \underline{C}_{g}$ is either set to the given values, or to $\min \left\{\bar{S}_{g}, \underline{S}_{g}\right\}$, whichever is the minimum. If no positive value is available, we set them to 1 . Likewise, missing $U T_{g}, D T_{g}$ are set to 1 . We assumed that the minimum up/downtime requirements are not restrictive at $t=0$, and generators incur cold start-up cost after 5 time periods. Generators with no cost entries are neglected (104 cases). Finally, we consider the realized demand dat ${ }^{2}$ of the PJM region between 01/31/2010 and 01/06/2010, where the former marks the date pertaining to the winter test problem.

\section{REFERENCES}

[1] B. Hobbs, M. Rothkopf, R. O'Neill, and H.-P. Chao, Eds., The Next Generation of Electric Power Unit Commitment Models, ser. Inter. Series in Oper. Res. \& Mgmt. Sci.. Springer-Verlag, 2001.

\footnotetext{
${ }^{1}$ http://www.ferc.gov/industries/electric/indus-act/market-planning/rtocommit-test.asp

${ }^{2}$ http://www.pjm.com/markets-and-operations/ops-analysis/historical-loaddata.aspx
} 
[2] R. P. O'Neill, T. Dautell, and E. Krall, "Recent ISO software enhancements and future software and modeling plans," FERC, Tech. Rep., 2011. [Online]. Available: http://www.ferc.gov/industries/electric/ indus-act/rto/rto-iso-soft-2011.pdf

[3] A. Turgeon, "Optimal unit commitment," IEEE Trans. Autom. Control, vol. 23 , no. 2 , pp. 223-227, 1977.

[4] C. K. Pang, G. B. Sheble, and F. Albuyeh, "Evaluation of dynamic programming based methods and multiple area representation for thermal unit commitments," IEEE Trans. Power App. Syst., vol. 100, no. 3, pp. $1212-1218,1981$.

[5] J. Muckstadt and S. A. Koenig, "An application of lagrangian relaxation to scheduling in power-generation systems," Oper. Res., vol. 25, no. 3, pp. 387-403, 1977.

[6] J. F. Bard, "Short-term scheduling of thermal-electric generators using lagrangian relaxation," Oper. Res., vol. 36, no. 5, pp. 756-766, 1988.

[7] C. Tseng, C. Li, and S. Oren, "Solving the unit commitment problem by a unit decommitment method," J. of Opt. Theory, vol. 105, pp. 707-730, 2000.

[8] N. Padhy, "Unit commitment - A bibliographical survey," IEEE Trans. Power Syst., vol. 19, no. 2, pp. 1196-1205, May 2004.

[9] B. Saravanan, S. Das, S. Sikri, and D. P. Kothari, "A solution to the unit commitment problem - a review," Frontiers in Energy, vol. 7, pp. 223-236, 2013.

[10] T. Li and M. Shahidehpour, "Price-based unit commitment: a case of lagrangian relaxation versus mixed integer programming," IEEE Trans. Power Syst., vol. 20, no. 4, pp. 2015-2025, 2005.

[11] D. Rajan and S. Takriti, "Minimum up / down polytopes of the unit commitment problem with start-up costs," IBM, Tech. Rep., 2005.

[12] C. Gentile, G. Morales-Espana, and A. Ramos, "A tight MIP formulation of the unit commitment problem with start-up and shut-down constraints," EURO Journal on Combinatorial Optimization, 2016.

[13] J. Ostrowski, M. F. Anjos, and A. Vannelli, "Tight mixed integer linear programming formulations for the unit commitment problem," IEEE Trans. Power Syst., vol. 27, no. 1, pp. 39-46, 2012.

[14] P. Damcı-Kurt, S. Küçükyavuz, D. Rajan, and A. Atamtürk, "A polyhedral study of production ramping," Math. Prog., vol. 158, no. 1, pp. 175-205, 2015.

[15] K. Cheung, D. Gade, C. Silva-Monroy, S. M. Ryan, J.-P. Watson, R. J.-B. Wets, and D. L. Woodruff, "Toward scalable stochastic unit commitment," Energy Systems, pp. 1-22, 2015.

[16] D. Bertsimas, E. Litvinov, X. A. Sun, J. Zhao, and T. Zheng, "Adaptive robust optimization for the security constrained unit commitment problem," IEEE Trans. Power Syst., vol. 28, no. 1, pp. 52-63, 2013.

[17] A. A. B. Pritsker, L. J. Waiters, and P. M. Wolfe, "Multiproject scheduling with limited resources: A zero-one programming approach," Mgmt. Sci., vol. 16, no. 1, pp. 93-108, 1969.

[18] D. Bertsimas, S. Gupta, and G. Lulli, "Dynamic resource allocation: A flexible and tractable modelling framework," European Journal of Operational Research, vol. 236, no. 1, pp. 14-26, 2014.

[19] L. L. Garver, "Power generation scheduling by integer programming development of theory," Power App. and Syst., Part III, Trans. of the Amer. Inst. of Elect. Eng., vol. 81, no. 3, pp. 730-734, 1962.

[20] M. Shahidehpour, H. Yamin, and Z. Li, Market Operations in Electric Power Systems: Forecasting, Scheduling, and Risk Management. IEEE, Wiley-Interscience, 2002.

[21] G. Morales-España, J. M. Latorre, and A. Ramos, "Tight and compact MILP formulation for the thermal unit commitment problem," IEEE Trans. Power Syst., vol. 28, no. 4, pp. 4897-4908, 2013.

[22] R. Baldick, "The generalized unit commitment problem," IEEE Trans. Power Syst., vol. 10, no. 1, pp. 465-475, 1995.

[23] M. Carrión and J. M. Arroyo, "A computationally efficient mixed-integer linear formulation for the thermal unit commitment problem," IEEE Trans. Power Syst., vol. 21, no. 3, pp. 1371-1378, 2006.

[24] J. M. Arroyo and A. J. Conejo, "Optimal response of a thermal unit to an electricity spot market," IEEE Trans. Power Syst., vol. 15, no. 3, pp. 1098-1104, 2000.

[25] S. Takriti and J. Birge, "Using integer programming to refine lagrangianbased unit commitment solutions," IEEE Trans. Power Syst., vol. 15, no. 1 , pp. 151-156, 2000

[26] J. Lee, J. Leung, and F. Margot, "Min-up / min-down polytopes," Discrete Optimization, vol. 1, pp. 77-85, 2004.

[27] R. Jiang, Y. Guan, and J. P. Watson, "Cutting planes for the multistage stochastic unit commitment problem," Math. Prog., vol. 157, no. 1, pp. 121-151, 2016.

[28] K. Pan and Y. Guan, "A polyhedral study of the integrated minimumup/-down time and ramping polytope," Optimization Online, 2016.
[29] M. P. Nowak and W. Römisch, "Stochastic Lagrangian relaxation applied to power scheduling in a hydro-thermal system under uncertainty," Ann. Oper. Res., vol. 100, no. 1-4, pp. 251-272, 2000.

[30] E. Krall, M. Higgins, and R. P. O'Neill, "RTO unit commitment test system," FERC, Tech. Rep., 2012. [Online]. Available: http: //www.ferc.gov/legal/staff-reports/rto-COMMITMENT-TEST.pdf

[31] J. Dupačová, N. Gröwe-Kuska, and W. Römisch, "Scenario reduction in stochastic programming," Math. Prog., vol. 95, no. 3, pp. 493-511, 2003.

[32] S. Sen, L. Yu, and T. Genc, "A stochastic programming approach to power portfolio optimization," Oper. Res., vol. 54, no. 1, pp. 55-72, 2006.

[33] New York ISO, "Power trends 2016: The changing energy landscape," Tech. Rep., 2016. [Online]. Available: http://www.nyiso.com/public/ webdocs/media_room/publications_presentations/Power_Trends/Power_ Trends/2016-power-trends-FINAL-070516.pdf

[34] G. L. Nemhauser and L. A. Wolsey, Integer programming and combinatorial optimization. New York: John Wiley and Sons, 1999.

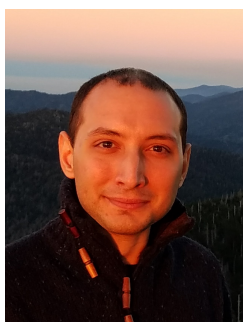

Semih Atakan received his B.Sc. degree in manufacturing systems engineering, and M.Sc. degree in industrial engineering from Sabanc1 University, Istanbul, Turkey, in 2010 and 2012, respectively.

$\mathrm{He}$ is currently a Ph.D. student at the University of Southern California, Los Angeles, CA. His main research interests are stochastic, convex, and mixedinteger optimization; risk measures; optimization applications, such as power systems planning, disaster management, and scheduling.

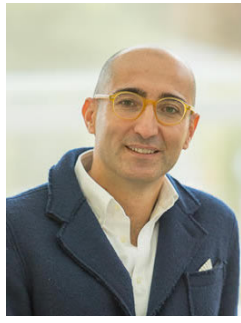

Guglielmo Lulli is Senior Lecturer in the Department of Management Science at Lancaster University. Prior to join Lancaster University, he was Assistant Professor at University of Milano-Bicocca. He earned his $\mathrm{PhD}$ in Operations Research at University of Rome "La Sapienza" in 2003. During his studies, he visited University of Maryland and University of Arizona, both for one year appointment. He received a Fulbright Fellowship at Massachusetts Institute of Technology, and the 2013 INFORMS TSL Best Published Paper award.

Lulli's research interests focus on deterministic and stochastic optimization particularly as applied to transportation and logistic operations and energy. He participated in several research projects financed by Italian, European and American agencies.

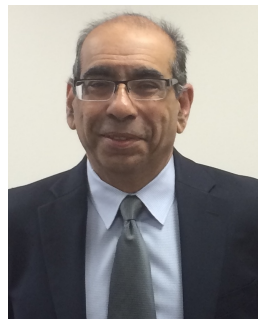

Suvrajeet Sen is a Professor in the Daniel J. Epstein Department of Industrial and Systems Engineering at the University of Southern California. Over the years, Professor Sen has held several other positions, including Professor at the University of Arizona, and a Program Director of Operations Research, as well as Service Enterprise Systems at the National Science Foundation (NSF). In the past few years he has led a group of world-renowned OR visionaries to identifying new themes of OR for problems motivated by Grand Challenges of the National Academy of Engineering. His research is devoted to many categories of optimization models, algorithms, and applications of stochastic programming problems. $\mathrm{He}$ is a Fellow of INFORMS. Recently he led a group which was awarded the 2015 INFORMS Computing Society Prize for their work on stochastic mixed-integer programming. 